\title{
Diagnostic Performance of MRI for Assessing Parametrial Invasion in Cervical Cancer: A Head-to-Head Comparison between Oblique and True Axial T2-Weighted Images
}

\author{
Sungmin Woo, $M D^{1}$, Min Hoan Moon, $M D^{2}$, Jeong Yeon Cho, $M D^{1,3}$, Seung Hyup Kim, $M D^{1,3}$, \\ Sang Youn Kim, MD ${ }^{1}$
}

${ }^{1}$ Department of Radiology, Seoul National University Hospital, Seoul National University College of Medicine, Seoul, Korea; ${ }^{2}$ Department of Radiology, Seoul Metropolitan Government, Seoul National University Boramae Medical Center, Seoul, Korea; ${ }^{3}$ Institute of Radiation Medicine and Kidney Research Institute, Seoul National University Medical Research Center, Seoul, Korea

Objective: To directly compare the diagnostic performance of true and oblique axial T2-weighted imaging (T2WI) for assessing parametrial invasion (PMI) in cervical cancer.

Materials and Methods: This retrospective study included 71 women with treatment-naive cervical cancer who underwent MRI that included both oblique and true axial T2WI, followed by radical hysterectomy. Two blinded radiologists (Radiologist 1 and Radiologist 2) independently assessed the presence of PMI on both sequences using a 5-point Likert scale. Receiver operating characteristic (ROC) curve analysis was performed, with a subgroup analysis for tumors sized $>2.5 \mathrm{~cm}$ and $\leq 2.5$ $\mathrm{cm}$ in diameter. Inter-reader agreement was assessed with kappa $(\mathrm{k})$ statistics.

Results: At hysterectomy, 15 patients (21.1\%) had PMI. For Radiologist 1, the area under the ROC curve (AUC) was greater for oblique axial than for true axial T2WI $\{0.941$ (95\% confidence interval [CI] $=0.858-0.983)$ vs. $0.917(95 \% \mathrm{CI}=0.827-$ $0.969), p=0.027\}$. The difference was not significant for Radiologist $2(0.879[95 \%$ CI $=0.779-0.944]$ vs. 0.827 [95\% CI $=0.719-0.906], p=0.153)$. For tumors $>2.5 \mathrm{~cm}$, AUC was greater with oblique than with true axial T2WI (0.906 vs. 0.860 , $p=0.046$ for Radiologist 1 and 0.839 vs. $0.765, p=0.086$ for Radiologist 2). Agreement between the radiologists was almost perfect for oblique axial T2WI $(k=0.810)$ and was substantial for true axial T2WI $(k=0.704)$.

Conclusion: Oblique axial T2WI potentially provides greater diagnostic performance than true axial T2WI for determining PMI, particularly for tumors $>2.5 \mathrm{~cm}$. The inter-reader agreement was greater with oblique axial T2WI.

Keywords: Cervical cancer; Magnetic resonance imaging; T2-weighted imaging; Parametrial invasion

\section{INTRODUCTION}

Cervical cancer is the second leading malignancy affecting women worldwide (1). The presence of parametrial invasion (PMI) affects prognosis and treatment decisions, and

Received May 8, 2018; accepted after revision August 29, 2018.

Corresponding author: Sang Youn Kim, MD, Department of Radiology, Seoul National University Hospital, Seoul National University College of Medicine, 101 Daehak-ro, Jongno-gu, Seoul 03080, Korea.

- Tel: (822) 2072-4897 - Fax: (822) 743-6385

- E-mail: iwishluv@empas.com

This is an Open Access article distributed under the terms of the Creative Commons Attribution Non-Commercial License (https://creativecommons.org/licenses/by-nc/4.0) which permits unrestricted non-commercial use, distribution, and reproduction in any medium, provided the original work is properly cited. therefore assessment of PMI is crucial in the pretreatment evaluation of cervical cancer $(2,3)$. Pretreatment staging of cervical cancer currently follows the revised International Federation of Gynecology and Obstetrics (FIGO) staging system, which is based on physical examination (4). However, physical examination results in relatively high rates of under- and over-staging of PMI, whereas metaanalyses have reported good performance with MRI for the assessment of PMI $(5,6)$; therefore, although MRI is not mandatory, the FIGO staging system recommends its use when available.

Recently, the European Society of Urogenital Radiology (ESUR) issued a guideline regarding the use of MRI for staging cervical cancer (7), stating that oblique axial T2weighted imaging (T2WI) is crucial for evaluating PMI. 
This is because the uterine cervix is often tilted (to the right or left, anteverted or retroverted), thus impeding accurate assessment of PMI using only axial images. However, evidence from original research on the increased value of using oblique axial T2WI for the assessment of PMI is lacking; the literature supporting the use of oblique sequences mostly comprises guidelines or review articles published by experts in the field (7-10). The purpose of this study, therefore, was to directly compare the diagnostic performance of true and oblique axial T2WI for the assessment of PMI in patients with cervical cancer.

\section{MATERIALS AND METHODS}

\section{Patient Population}

This study was approved by our Institutional Review Board, with the requirement for informed consent waived because of the retrospective design. A computerized search of our radiologic database and electronic medical records identified patients diagnosed with cervical cancer who underwent pelvic MRI examinations between July 2015 and December 2016. The start date of the search period was chosen as the time that MRI of the cervix using both true and oblique axial T2WI commenced at our institution. The initial search yielded 182 consecutive patients, among whom 72 met the following inclusion criteria: 1) oblique

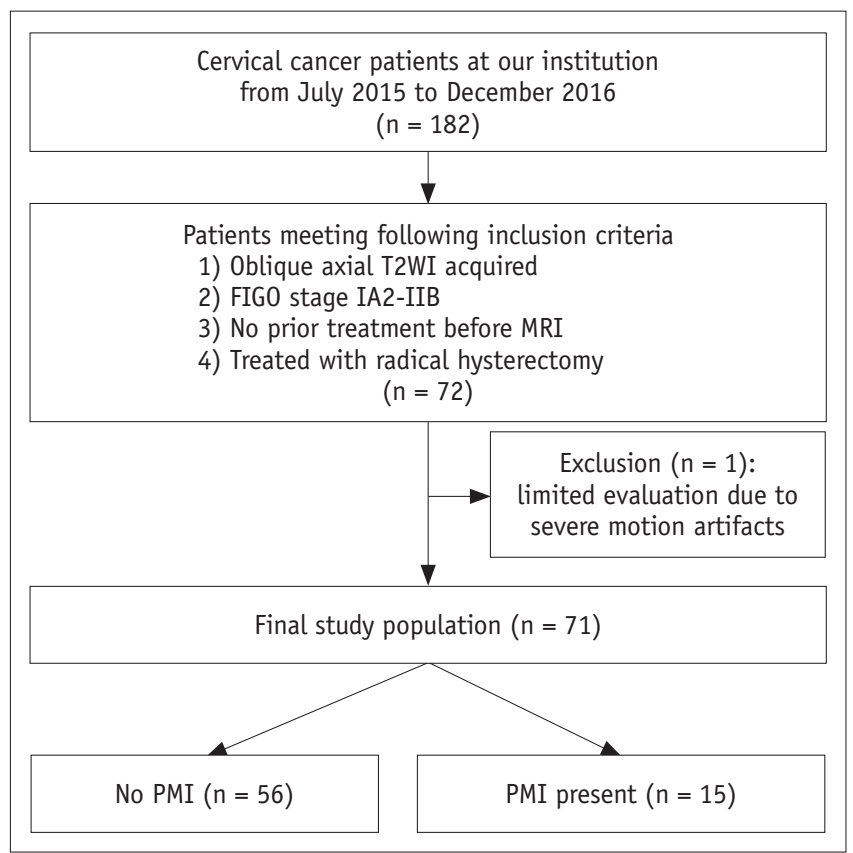

Fig. 1. Flowchart of patient selection process. $F I G 0=$ International Federation of Gynecology and Obstetrics, PMI = parametrial invasion, T2WI = T2-weighted imaging axial T2WI was performed; 2) the patient was classified as FIG0 stage IA2-IIB; 3) no treatment of the cervix had been administered prior to MRI, including surgery, chemotherapy, and radiotherapy; and 4) the patient underwent definitive treatment with radical hysterectomy. Of these 72 patients, one was excluded from the analysis because of severe motion artifacts that resulted in a limited evaluation of PMI. Ultimately, 71 women were included in this study. Figure 1 shows the patient selection process.

\section{MRI Protocol}

MRI scans were acquired using various 1.5-tesla and 3-tesla scanners with phased-array body coils. Before image acquisition, $20 \mathrm{mg}$ of hyoscine butyl bromide (Buscopan; Boehringer Ingelheim Pharma, Ingelheim am Rhein, Germany) was injected intramuscularly to suppress motion artifacts due to peristalsis. Our MRI protocol for the assessment of cervical cancer consisted of T2WI of the pelvis in four planes (true axial, axial oblique, coronal, and sagittal), axial T1-weighted imaging (T1WI) of the pelvis, diffusion-weighted imaging with the corresponding apparent diffusion coefficient maps of the pelvis, dynamic contrast-enhanced MRI of the pelvis, and T1WI of the upper abdomen. Prior to commencing the acquisition of oblique axial T2WI at our institution, the MRI technicians who perform cervical MRI were trained in this mode of acquisition by a staff radiologist subspecialized in gynecologic imaging. Thereafter, routine MRI scanning was performed by the MRI technicians without supervision. Specifically, oblique axial T2WI was performed using the "double oblique" technique, which correctly aligns the plane along the true cervical axis by angling off both sagittal and coronal planes based on scout images (10).

The following sequences among those included in our multiparametric MRI protocol were used in this study: 1) true and oblique axial T2WI for assessment of PMI, 2) both T2WI types plus coronal/sagittal T2WIs and diffusionweighted imaging for primary tumor localization, and 3) T2WI in three orthogonal planes for measurement of tumor size. The detailed MRI parameters are shown in Table 1.

\section{Image Analysis}

All images were assessed by two independent radiologists (with 6 and 19 years of post-fellowship experience in gynecologic imaging, respectively). The radiologists, hereafter referred to as R1 and R2, were aware that the patients had pathologically proven cervical cancer but 
Table 1. MRI Parameters for 1.5- and 3-tesla Scanners

\begin{tabular}{|c|c|c|c|c|}
\hline \multirow{2}{*}{ Imaging Plane } & \multicolumn{2}{|c|}{ 1.5-tesla* } & \multicolumn{2}{|c|}{ 3-tesla ${ }^{\dagger}$} \\
\hline & Axial & Axial Oblique & Axial & Axial Oblique \\
\hline Field of view (mm) & $\begin{array}{c}220 \times 220,240 \times 240 \\
250 \times 250\end{array}$ & $\begin{array}{c}220 \times 220,240 \times 240 \\
280 \times 280\end{array}$ & $220 \times 220$ & $\begin{array}{c}220 \times 220,223 \times 223 \\
300 \times 300\end{array}$ \\
\hline Matrix size & $338-384 \times 224$ & $338-384 \times 224$ & $448 \times 312-403$ & $448 \times 312-403$ \\
\hline Slice thickness/gap (mm) & $5 / 0-1$ & $5 / 0$ & $5 / 0-0.5$ & $5 / 0$ \\
\hline TR/TE (ms) & $2400-7600 / 104.2-108.2$ & $3050-7983.3 / 105.7-111.3$ & $2880-4516 / 90-102.4$ & $2880-4664 / 90-104.2$ \\
\hline Number of signals averaged & 2 & 2 & $1-2$ & $1-2$ \\
\hline
\end{tabular}

${ }^{*}$ 3-tesla scanners included MR750W (GE Healthcare), Ingenia (Philips Healthcare), and Verio and Skyra (Siemens Healthineers), ${ }^{\dagger} 1.5$-tesla scanners included Signa HDxt and Signa Excite HD (GE Healthcare). TE = echo time, TR = repetition time

were blinded to all other clinical information, including any finding of PMI based on histopathological assessment of surgical specimens. Cervical cancer was defined as a focal cervical lesion on T2WI with a higher signal intensity than that of the adjacent normal myometrium and that corresponded to an area with high signal intensity on diffusion-weighted imaging $\left(b=800 \mathrm{~s} / \mathrm{mm}^{2}\right.$ or $1000 \mathrm{~s} /$ $\mathrm{mm}^{2}$ ) (11). The two radiologists independently assessed and recorded the likelihood of PMI based on each imaging sequence using a 5-point Likert scale, as follows: 1 , definitely no PMI; 2, probably no PMI; 3, possible PMI; 4, PMI probably present; and 5, PMI definitely present. The diagnostic criteria for PMI on true and oblique axial T2WI were based on a comprehensive assessment of the following well-established imaging features: full-thickness disruption of the normal cervical stroma (which shows as a hypointense signal on T2WI), a spiculated interface between the cervical cancer and parametrium, soft tissue extending into the parametrium, or encasement of the periuterine vessels (12). To avoid recall bias, the two interpretation sessions (for true axial and oblique axial T2WI) were separated by a 6-week interval and were performed by the radiologists in random order (13).

\section{Clinical and Pathologic Data Collection}

We searched our electronic medical records and pathology database for the clinicopathologic findings of the included patients. The data collected included age, clinical FIG0 staging recorded by the attending gynecologist, histologic subtype, and the presence of PMI on surgical specimens.

\section{Statistical Analysis}

The statistical analysis was performed using SPSS, version 21.0 (IBM Corp., Armonk, NY, USA) and MedCalc version 12.3.0.0 (MedCalc Software, Mariakerke, Belgium). The patients were categorized into two groups based on the presence or absence of PMI on surgical specimens. Receiver operating characteristic (ROC) curve analysis was used to assess the diagnostic performance of true and oblique axial T2WI for the prediction of PMI, and the two planes were compared in terms of the area under the ROC curve (AUC). In a subgroup analysis, the tumors were divided into groups according to diameters of $>2.5 \mathrm{~cm}$ and $\leq 2.5 \mathrm{~cm}$ (14). The inter-reader agreement between R1 and R2 was evaluated using weighted kappa $(\mathrm{k})$ statistics with linear weights (15). The degree of agreement was interpreted according to the following categories for $k$ values (16): 0.00-0.20, slight; $0.21-0.40$, fair; $0.41-0.60$, moderate; $0.61-0.80$, substantial; and $0.81-1.00$, almost perfect. Twosided $p$ values $<0.05$ were considered to indicate statistical significance.

\section{RESULTS}

The baseline characteristics of the study population are summarized in Table 2. The median age of the 71 included women was 52 years (mean \pm standard deviation [SD], $50.8 \pm 11.4$ years; age range, $27-74$ years). Their FIG0 stages ranged from IA to IIB. The predominant histological subtype was squamous cell carcinoma (55 of $71[77.5 \%]$ ). PMI was present on radical hysterectomy specimens in 15 $(21.1 \%)$ of the patients. The median diameter of the tumors on MRI was $3.0 \mathrm{~cm}$ (mean $\pm \mathrm{SD}, 2.9 \pm 1.9 \mathrm{~cm}$; interquartile range, $1.3-4.4 \mathrm{~cm}$ ). The median interval between MRI and surgery was 11 days (interquartile range, 6-17 days).

Figure 2 shows the ROC analysis of the diagnostic performance of the true and oblique axial T2WI for the prediction of PMI. For R1, the diagnostic performance of oblique axial T2WI was significantly better than that of true axial T2WI, as measured by the AUCs: 0.941 (95\% confidence interval [CI], 0.858-0.983) vs. 0.917 (95\% CI, $0.827-0.969)$, respectively $(p=0.027)$. For R2, although 
the AUC of oblique axial T2WI was greater than that of true axial T2WI (0.879 [95\% CI, 0.779-0.944] vs. 0.827 [95\% CI, 0.719-0.906]), the difference was not statistically significant $(p=0.153)$. Figure 3 shows the assessment of PMI based on oblique and true axial T2WI for a representative case.

In the subgroup analysis, the tumors of 38 patients were $>2.5 \mathrm{~cm}$ in diameter, and those of 33 patients were $\leq 2.5$ $\mathrm{cm}$. ROC curve analysis could not be performed for tumors $\leq 2.5 \mathrm{~cm}$ because PMI was found in the surgical specimen of only one $(3.0 \%)$ of these patients. Of the 38 patients

\section{Table 2. Baseline Characteristics}

\begin{tabular}{|c|c|}
\hline Variable & Data \\
\hline Total no. of patients & 71 \\
\hline Median age $(y)^{*}$ & $52(27-74)$ \\
\hline \multicolumn{2}{|l|}{ Clinical FIG0 stage ${ }^{\dagger}$} \\
\hline IA & $11(15.5)$ \\
\hline IB1 & $35(49.3)$ \\
\hline IB2 & $9(12.7)$ \\
\hline IIA & $12(16.9)$ \\
\hline IIB & $4(5.6)$ \\
\hline \multicolumn{2}{|l|}{ Histologic subtype $^{\dagger}$} \\
\hline $\mathrm{SqCC}$ & $55(77.5)$ \\
\hline Other & $16(22.5)$ \\
\hline PMI on surgical specimen ${ }^{\dagger}$ & $15(21.1)$ \\
\hline Median tumor size on MRI $(\mathrm{cm})^{\ddagger}$ & $3.0(1.3-4.4)$ \\
\hline Median interval between MRI and surgery $(\mathrm{d})^{\dagger}$ & $11(6-17)$ \\
\hline
\end{tabular}

Data in parentheses are * Range, ${ }^{\dagger}$ Percentage, or ${ }^{\ddagger}$ Interquartile range. FIGO = International Federation of Gynecology and Obstetrics, $\mathrm{PMI}=$ parametrial invasion, $\mathrm{SqCC}=$ squamous cell carcinoma with tumors $>2.5 \mathrm{~cm}, 14$ (37\%) were found to have PMI on pathology. For R1, the diagnostic performance of oblique axial T2WI was significantly better than that of true axial T2WI, as shown by the AUCs: 0.906 (95\% CI, 0.767-0.976) vs. 0.860 (95\% CI, 0.709-0.951), respectively ( $p=0.046)$. For R2, the AUC of oblique axial T2WI was greater than that

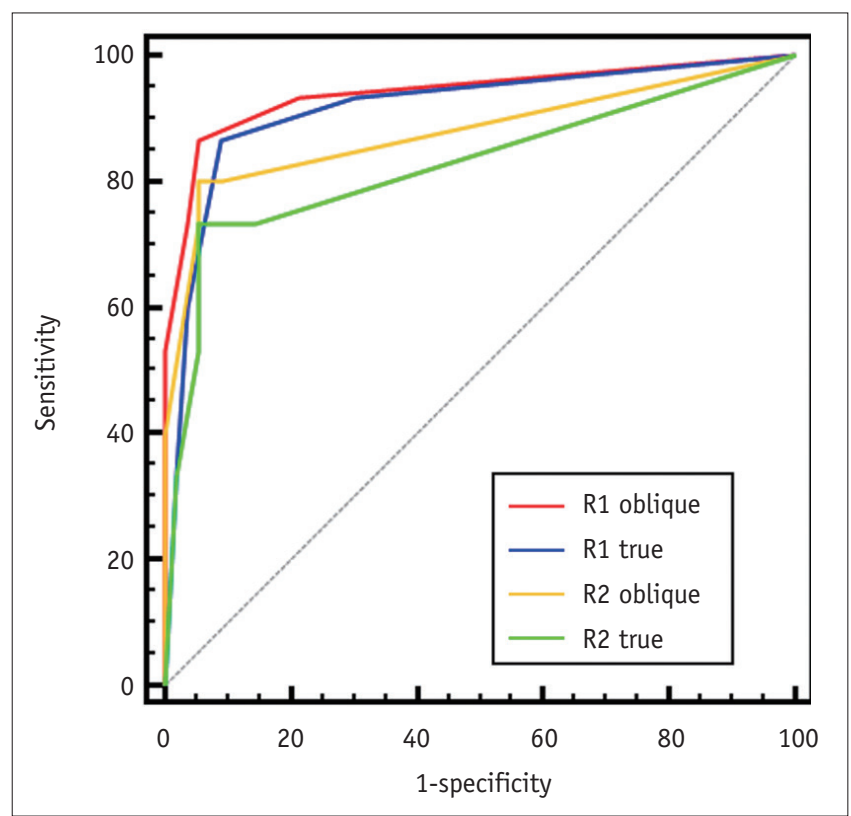

Fig. 2. ROC curve analysis for entire study population. In assessments performed by Radiologist 1, AUC was significantly greater for oblique axial T2WI than for true axial T2WI (0.941 [95\% CI, 0.8580.983 ] vs. 0.917 [ $95 \%$ CI, 0.827-0.969], $p=0.027)$. In assessments performed by Radiologist 2, difference in AUC was not significant (0.879 [95\% CI, 0.779-0.944] vs. 0.827 [95\% CI, 0.719-0.906], $p$ $=0.153) . A U C=$ area under curve, $\mathrm{CI}=$ confidence interval, $\mathrm{ROC}=$ receiver operating characteristic

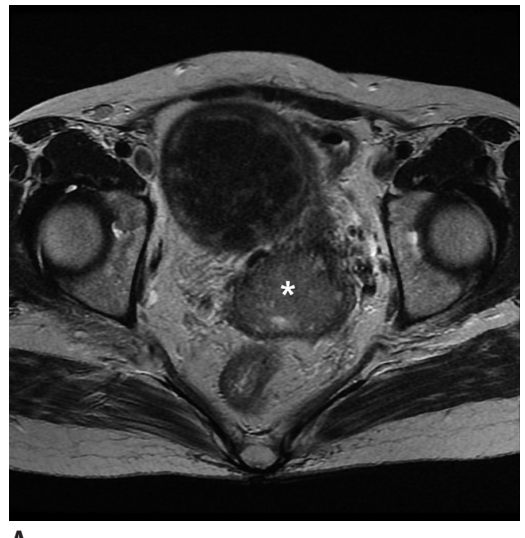

A

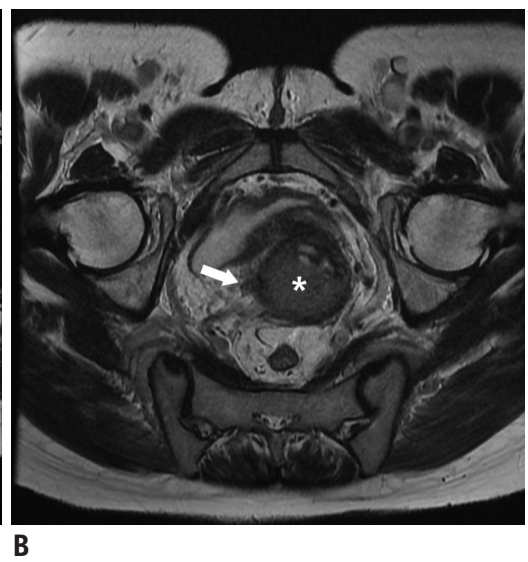

B

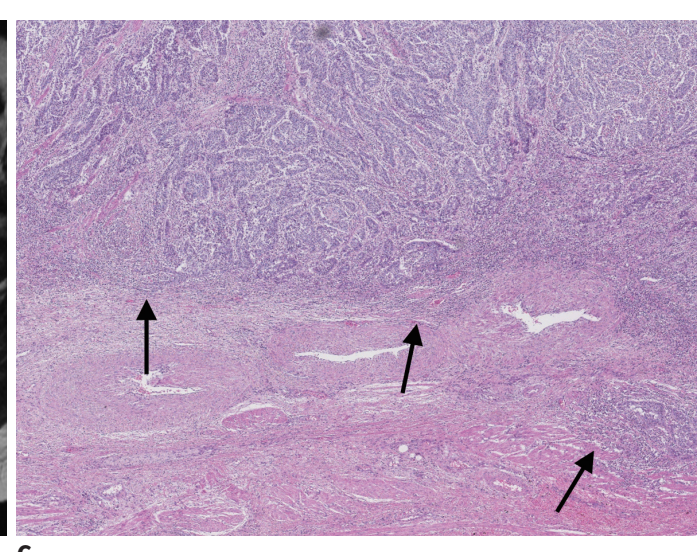

C

Fig. 3. T2WI of 50-year-old woman with biopsy-proven clinical FIG0 stage IIA1 cervical cancer, with PMI after surgery.

A. True axial plane. B. Oblique axial plane. C. Microscopic image (H\&E staining, magnification, x 40). Cervical mass was observed (asterisk). Small nodular soft tissue in right parametrium was better demonstrated on oblique axial T2WI. Using 5-point Likert scale, both radiologists assigned scores of 3 (possible PMI) to true axial T2WI and 4 (PMI probably present) to oblique axial T2WI. Patient underwent radical hysterectomy with bilateral salpingo-oophorectomy, and pelvic and para-aortic lymph node dissection. Histopathological assessment revealed invasive squamous cell carcinoma with right-sided PMI. On histopathological analysis of microscopic slides, PMI was noted (arrows). 


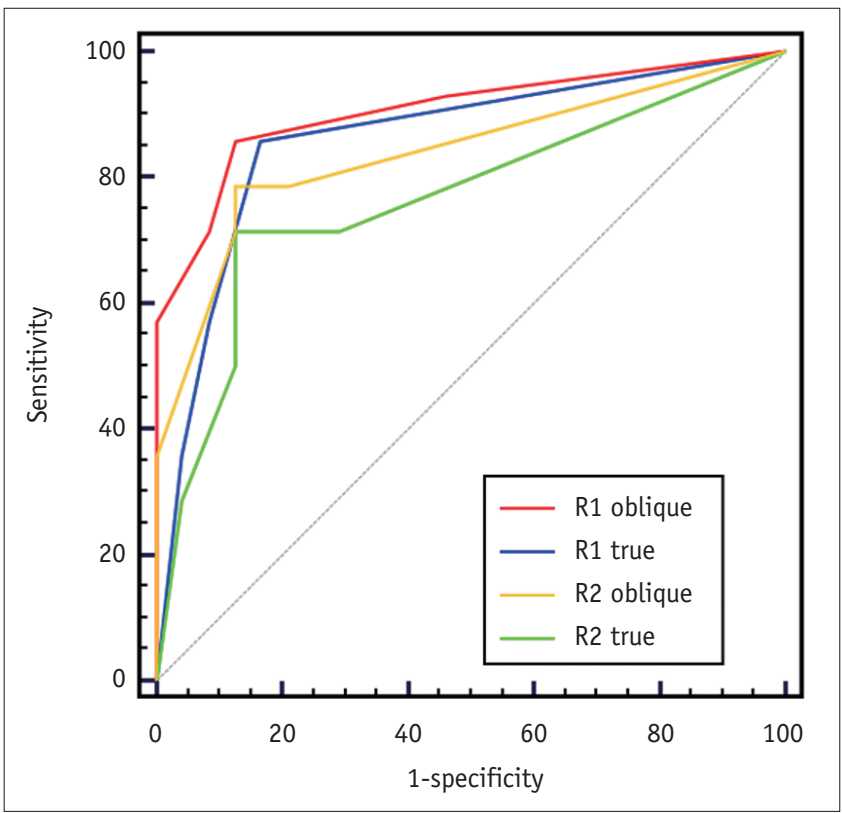

Fig. 4. ROC curve analysis for tumors sized $>2.5 \mathrm{~cm}$. In assessments performed by both radiologists, AUC was greater for oblique axial T2WI than for true axial T2WI. Difference was statistically significant for Radiologist 1 (0.906 [95\% CI, 0.767-0.976] vs. 0.860 [95\% CI, 0.709-0.951], $p=0.046)$ and borderline significant for Radiologist 2 (0.839 [95\% CI, 0.684-0.938] vs. 0.765 [95\% CI, $0.599-0.887], p=0.086$ ).

of true axial T2WI with borderline statistical significance: 0.839 (95\% CI, 0.684-0.938) vs. 0.765 (95\% CI, 0.599$0.887)$, respectively $(p=0.086)$. Figure 4 presents the ROC curves of true and oblique axial T2WI assessed by the two radiologists for the prediction of PMI.

The inter-reader agreement was classed as almost perfect for the interpretation of oblique axial T2WI, with a $k$ of 0.810 (95\% CI, 0.723-0.896), and as substantial for true axial T2WI, with a $k$ of 0.704 (95\% CI, 0.610-0.798).

\section{DISCUSSION}

In this study, we performed a head-to-head comparison between oblique and true axial T2WI with two independent radiologists assessing PMI in patients with cervical cancer. Oblique axial T2WI resulted in higher AUC values than true axial T2WI in the assessments of both radiologists; the difference was statistically significant for one radiologist but not the other. Although not conclusive, this suggests that oblique axial T2WI may be superior to true axial T2WI for diagnostic performance. This is plausible because the uterine cervix is typically tilted, resulting in partial volume effects between cervical cancer and adjacent stromal tissue on true axial T2WI (10). In theory, adjusting the image acquisition plane could overcome this issue. Our study results are consistent with the literature supporting the use of oblique axial T2WI, which mostly comprises guidelines such as the ESUR guidelines (7) or review articles published by experts (8-10). To the best of our knowledge, only one original study has been conducted; Shiraiwa et al. (17) reported that oblique axial T2WI produced more accurate assessments than true axial T2WI (accuracy of 0.89 vs. 0.79 , $p=0.002$ ). However, it should be noted that this was based on a comparison between "thin-section" oblique axial T2WI (with a slice thickness of 3-5 mm) and "conventional" true axial T2WI (with a slice thickness of $8 \mathrm{~mm}$ ). By contrast, we used an identical $5-\mathrm{mm}$ slice thickness for both imaging planes, representing a more reliable "head-to-head" comparison between oblique and true axial T2WI.

The subgroup analysis based on tumor size on MRI resulted in two critical findings. First, tumor size appeared to be a major predictor of PMI, as has been consistently reported in the literature. Several size thresholds, including $2,2.5$, and $4 \mathrm{~cm}$, have been suggested for identifying a low-risk group for PMI $(14,18,19)$. In our study, in the subgroup of 33 patients with a tumor sized $\leq 2.5 \mathrm{~cm}$ on MRI, only one patient experienced PMI. This is in agreement with a previous study by Kamimori et al. (20), which reported that PMI was not observed in any tumors $<2 \mathrm{~cm}$ in diameter. Second, the greater value of oblique axial T2WI appeared to be more pronounced in the subgroup with tumors sized > $2.5 \mathrm{~cm}$. Although the difference in diagnostic performance between oblique and true axial T2WI for the entire study population was only significant for R1 $(p=0.027)$ and not for R2 ( $p=0.153)$, in the subgroup with tumors $>2.5 \mathrm{~cm}$, the difference was statistically significant for $\mathrm{R} 1(p=0.046)$ and borderline significant for $\mathrm{R} 2(p=0.086)$.

Although the results of our study demonstrated the potentially greater value of oblique axial T2WI for evaluating PMI, several factors may explain why the difference for R2 was not statistically significant and why the degree of difference was not marked for either radiologist (AUC = 0.941 vs. 0.917 for R1 and 0.879 vs. 0.827 for R2). First, the study included a relatively small number of patients $(n=$ 71) and the difference between oblique and true axial T2WI may have proved significantly different for both R1 and R2 with a larger study population, particularly for patients with tumors $>2.5 \mathrm{~cm}$. Second, although we performed a "headto-head" comparison between oblique and true axial T2WI, the tumor localization accounted for multiplanar (coronal and sagittal) T2WI and axial diffusion-weighted imaging. 
This may have resulted in overestimation of the diagnostic performance as compared with assessing only oblique or true axial T2WI, thereby diminishing the difference between the two planes. A recent meta-analysis by Woo et al. (6) that reviewed the diagnostic performance of MRI for detection of PMI in cervical cancer revealed that whether a study included both oblique axial and sagittal T2WI in the protocol was not a source of heterogeneity among the reviewed studies. Similarly, Fridsten et al. (21) investigated the utility of oblique axial imaging, but compared two sets of MRI protocols (true axial T2WI + sagittal T2WI vs. true axial T2WI + sagittal T2WI + oblique axial T2WI) and found that T stage was minimally altered and in only $7 \%(4 / 57)$ of patients. As with our study, this may have been because all 14 studies included in the meta-analysis and the study by Fridsten et al. (21) acquired T2WI in at least two imaging planes regardless of the presence or absence of oblique axial T2WI. Third, the level of experience could have affected the differences in the results obtained by the two radiologists. Previous studies have shown that level of experience may change tumor staging and alter patient management (22, 23). Taking into consideration these potential reasons, we believe that future studies with a larger study population and a greater number of radiologists (with various levels of experience) in a multicenter setting, with oblique axial planes acquired under radiologist supervision may provide conclusive evidence of the relative values of oblique axial and true axial T2WI for assessing PMI.

The inter-reader agreement was greater with oblique axial T2WI than with true axial T2WI, which were found to be almost perfect $(k=0.810)$ and substantial $(k=$ 0.704 ), respectively. This may have been because partial volume effects in the true axial T2WI led to results with greater discrepancy, whereas oblique axial T2WI acquired perpendicular to the cervical axis would be relatively free of such effects, resulting in higher concordance between the radiologists. This may have profound clinical implications; for any imaging modality or method to be accepted by radiologists and referring clinicians, it must not only be accurate, but also reproducible. The higher inter-reader agreement for oblique axial T2WI is another reason for its use in the assessment of PMI.

Some limitations of our study warrant mention. First, the small number of patients and the retrospective study design may have introduced bias. Second, although the two image sets were presented in random order and separated by 6 weeks, the radiologists could not be truly blinded to the imaging plane because any radiologist would immediately recognize whether an image was acquired in the oblique axial or true axial plane. This may have biased the radiologist toward better diagnostic performance with the oblique axial T2WI. However, true blinding to the imaging plane is impossible unless the tumor and the immediately surrounding structure are cropped to exclude areas such as the pelvic bones, rectum, bladder, and pelvic vessels. Third, we did not perform subgroup analysis stratified to relevant factors such as magnetic field strength (1.5 tesla vs. 3 tesla) and different patterns of PMI. For instance, previous investigators have noted that using 3-tesla scanners could result in an improved signal-to-noise ratio and contrast-to-noise-ratio. Additional studies will be needed to elucidate the effects of such factors (24). Finally, despite the potential advantage of directly obtaining oblique axial planes prospectively, advances in technology allow for retrospective reconstruction into oblique planes from three-dimensional isotropic images initially acquired in other orthogonal planes (i.e., axial or sagittal) with the advantages of shorter acquisition time and relatively equal overall image quality $(25,26)$.

In conclusion, oblique axial T2WI seemed to provide potentially better diagnostic performance compared with true axial T2WI for the determination of PMI in patients with cervical cancer. The use of oblique axial T2WI may be of incremental value in patients with tumors $>2.5 \mathrm{~cm}$ in diameter. In addition, the inter-reader agreement was greater with oblique axial than with true axial T2WI.

\section{Conflicts of Interest}

The authors have no potential conflicts of interest to disclose.

\section{ORCID iDs}

Sang Youn Kim

https://orcid.org/0000-0003-4038-7345

Sungmin Woo

https://orcid.org/0000-0001-8459-8369

Min Hoan Moon

https://orcid.org/0000-0003-2448-2895

Jeong Yeon Cho

https://orcid.org/0000-0001-8659-0918

Seung Hyup Kim

https://orcid.org/0000-0002-9339-5907 


\section{REFERENCES}

1. Siegel R, Naishadham D, Jemal A. Cancer statistics, 2012. CA Cancer J Clin 2012;62:10-29

2. Landoni F, Bocciolone L, Perego P, Maneo A, Bratina G, Mangioni C. Cancer of the cervix, FIGO stages IB and IIA: patterns of local growth and paracervical extension. Int $\mathrm{J}$ Gynecol Cancer 1995;5:329-334

3. Peters WA 3rd, Liu PY, Barrett RJ 2nd, Stock RJ, Monk BJ, Berek JS, et al. Concurrent chemotherapy and pelvic radiation therapy compared with pelvic radiation therapy alone as adjuvant therapy after radical surgery in high-risk early-stage cancer of the cervix. J Clin Oncol 2000;18:1606-1613

4. Pecorelli S, Benedet JL, Creasman WT, Shepherd JH. FIGO staging of gynecologic cancer. 1994-1997 FIG0 Committee on Gynecologic Oncology. International Federation of Gynecology and Obstetrics. Int J Gynaecol Obstet 1999;65:243-249

5. Thomeer MG, Gerestein C, Spronk S, van Doorn HC, van der Ham E, Hunink MG. Clinical examination versus magnetic resonance imaging in the pretreatment staging of cervical carcinoma: systematic review and meta-analysis. Eur Radiol 2013;23:2005-2018

6. Woo S, Suh CH, Kim SY, Cho JY, Kim SH. Magnetic resonance imaging for detection of parametrial invasion in cervical cancer: an updated systematic review and meta-analysis of the literature between 2012 and 2016. Eur Radiol 2018;28:530-541

7. Balleyguier C, Sala E, Da Cunha T, Bergman A, Brkljacic B, Danza $F$, et al. Staging of uterine cervical cancer with MRI: guidelines of the European Society of Urogenital Radiology. Eur Radiol 2011;21:1102-1110

8. Sala E, Rockall AG, Freeman SJ, Mitchell DG, Reinhold C. The added role of MR imaging in treatment stratification of patients with gynecologic malignancies: what the radiologist needs to know. Radiology 2013;266:717-740

9. Sala E, Wakely S, Senior E, Lomas D. MRI of malignant neoplasms of the uterine corpus and cervix. AJR Am J Roentgenol 2007;188:1577-1587

10. Rauch GM, Kaur H, Choi H, Ernst RD, Klopp AH, Boonsirikamchai $\mathrm{P}$, et al. Optimization of MR imaging for pretreatment evaluation of patients with endometrial and cervical cancer. Radiographics 2014;34:1082-1098

11. Park JJ, Kim CK, Park SY, Park BK, Kim B. Value of diffusionweighted imaging in predicting parametrial invasion in stage IA2-IIA cervical cancer. Eur Radiol 2014;24:1081-1088

12. Sala E, Micco M, Burger IA, Yakar D, Kollmeier MA, Goldman $D A$, et al. Complementary prognostic value of pelvic magnetic resonance imaging and whole-body fluorodeoxyglucose positron emission tomography/computed tomography in the pretreatment assessment of patients with cervical cancer. Int J Gynecol Cancer 2015;25:1461-1467

13. Das M, Mühlenbruch G, Mahnken AH, Flohr TG, Gündel L, Stanzel S, et al. Small pulmonary nodules: effect of two computer-aided detection systems on radiologist performance.
Radiology 2006;241:564-571

14. Lee JY, Youm J, Kim TH, Cho JY, Kim MA, Suh DH, et al. Preoperative MRI criteria for trials on less radical surgery in stage IB1 cervical cancer. Gynecol Oncol 2014;134:47-51

15. McCarville MB, Kaste SC, Hoffer FA, Khan RB, Walton RC, Alpert BS, et al. Contrast-enhanced sonography of malignant pediatric abdominal and pelvic solid tumors: preliminary safety and feasibility data. Pediatr Radiol 2012;42:824-833

16. Landis JR, Koch GG. The measurement of observer agreement for categorical data. Biometrics 1977;33:159-174

17. Shiraiwa M, Joja I, Asakawa T, Okuno K, Shibutani 0, Akamatsu $\mathrm{N}$, et al. Cervical carcinoma: efficacy of thin-section oblique axial T2-weighted images for evaluating parametrial invasion. Abdom Imaging 1999;24:514-519

18. Lee JY, Youm J, Kim JW, Cho JY, Kim MA, Kim TH, et al. Identifying a low-risk group for parametrial involvement in microscopic stage IB1 cervical cancer using criteria from ongoing studies and a new MRI criterion. BMC Cancer 2015;15:167

19. Kong TW, Piao X, Chang SJ, Paek J, Lee Y, Lee EJ, et al. A predictive model for parametrial invasion in patients with FIGO stage IB cervical cancer: individualized approach for primary treatment. Int J Gynecol Cancer 2016;26:184-191

20. Kamimori T, Sakamoto K, Fujiwara K, Umayahara K, Sugiyama $\mathrm{Y}$, Utsugi $\mathrm{K}$, et al. Parametrial involvement in FIGO stage IB1 cervical carcinoma diagnostic impact of tumor diameter in preoperative magnetic resonance imaging. Int $\mathrm{J}$ Gynecol Cancer 2011;21:349-354

21. Fridsten S, Hellström AC, Hellman K, Sundin A, Söderén B, Blomqvist L. Preoperative MR staging of cervical carcinoma: are oblique and contrast-enhanced sequences necessary? Acta Radiol Open 2016;5:2058460116679460

22. Woo S, Kim SY, Cho JY, Kim SH. Assessment of deep myometrial invasion of endometrial cancer on MRI: added value of secondopinion interpretations by radiologists subspecialized in gynaecologic oncology. Eur Radiol 2017;27:1877-1882

23. Lakhman Y, D’Anastasi M, Miccò M, Scelzo C, Vargas HA, Nougaret $S$, et al. Second-opinion interpretations of gynecologic oncologic MRI examinations by sub-specialized radiologists influence patient care. Eur Radiol 2016;26:2089-2098

24. Hori M, Kim T, Murakami T, Imaoka I, Onishi H, Tomoda K, et al. Uterine cervical carcinoma: preoperative staging with 3.0T MR imaging--comparison with 1.5-T MR imaging. Radiology 2009;251:96-104

25. Park HJ, Lee SY, Choi SH, Ahn JH, Park SJ, Park JH, et al. Comparison of oblique coronal images in knee of threedimensional isotropic $\mathrm{T}_{2}$-weighted turbo spin echo MRI versus two-dimensional fast spin echo $\mathrm{T}_{2}$-weighted sequences for evaluation of posterior cruciate ligament injury. Br J Radiol 2016;89:20160554

26. Shin YR, Rha SE, Choi BG, Oh SN, Park MY, Byun JY. Uterine cervical carcinoma: a comparison of two- and three-dimensional T2-weighted turbo spin-echo MR imaging at 3.0 T for image quality and local-regional staging. Eur Radiol 2013;23:1150-1157 